\title{
Resolution of Chronic Headache Following Pineal Cyst Fenestration. Case Report
}

\author{
Erica Darian-Smith ${ }^{1,2^{*}}$, Chien Yew Kow ${ }^{1}$, Patrick Kwan ${ }^{3}$ and Jeffrey V. Rosenfeld ${ }^{1,4}$ \\ ${ }^{1}$ Department of Neurosurgery, Alfred Hospital, 55 Commercial Road, Melbourne, Victoria 3004, Australia \\ ${ }^{2}$ School of Medicine, University of Tasmania, Level 1, Medical Science 1, 17Liverpool Street, Hobart, Tasmania 7000, Australia \\ ${ }^{3}$ Departments of Medicine and Neurology, The University of Melbourne, RoyalMelbourne Hospital, 300 Grattan St, Parkville VIC 3050, Australia \\ ${ }^{4}$ Department Surgery, Central Clinical School, Monash University, Wellington Rd, Clayton VIC 3800, Australia
}

'Correspondence to:

Erica Darian-Smith, MBBS

Department of Neurosurgery, Alfred Hospital 55

Commercial Road, Melbourne

Victoria 3004, Australia

E-mail: edar2754@gmail.com

Received: February 06, 2017

Accepted: March 31, 2017

Published: April 04, 2017

Citation: Darian-Smith E, Kow CY, Kwan P, Rosenfeld JV. 2017. Resolution of Chronic Headache Following Pineal Cyst Fenestration. Case Report. J Neurol Exp Neurosci 3(1): 15-17.

Copyright: (C) 2017 Darian-Smith et al. This is an Open Access article distributed under the terms of the Creative Commons Attribution 4.0 International License (CC-BY) (http:// creativecommons.org/licenses/by/4.0/) which permits commercial use, including reproduction, adaptation, and distribution of the article provided the original author and source are credited.

Published by United Scientific Group

\section{Abstract}

Objective: We report a single case of resolution of chronic incapacitating headache following surgical fenestration of a large pineal cyst.

Background: There is an established link between pineal cysts and headaches particularly in the presence of ventriculomegaly and gaze paresis. Our patient's chronic headache was attributed to a large pineal cyst.

Result: Following surgical fenestration there was immediate, complete relief of the patient's unremitting headache. Ten months since the operation the patient remains headache free.

Conclusion: This case demonstrates that surgical intervention may play a role in management of patients who have large benign pineal cysts and intractable headache despite the absence of ventriculomegaly and gaze paresis.

\section{Keywords}

Headache, Pineal cyst fenestration, Chronic headache, Pineal cyst

\section{Introduction}

Pineal cysts, whilst usually benign and stable in size, may cause symptoms if greater than $15 \mathrm{~mm}$ [1]. Symptoms are generally non-specific and may include headache, fatigue, visual disturbance, vertigo and nausea. With a female predilection, prevalence ranges from $1-1.5 \%$ in the general population centered on large magnetic resonance imaging studies. Natural evolution suggests growth of pineal cysts during adolescence with slow regression thereafter. For these asymptomatic patients continued surveillance and surgical intervention is not indicated. Patients with pineal cysts may fall into three categories: asymptomatic, symptomatic in the presence of radiological mass effect and lastly, symptomatic in the absence of radiologically detected mass effect - as in this case. For the latter cohort, management options and guidelines are more uncertain [2].

\section{Case}

A 32-year-old female presented with a ten-year history of intermittent, stress-related, generalized headache, with each episode lasting one to two days. In early 2015 duration and intensity of patient's headaches increased, with the patient reporting constant incapacitating daily headache for a period of two months. Magnetic resonance imaging (MRI) of her brain showed an ovoid pineal cyst, with a benign appearance, but was otherwise reported as normal. 
In February 2016, the headaches continued to occur multiple days weekly. The character was described as a pressure, bandlike sensation, which was distributed bitemporally. There were no preceding factors, the headache was not postural in nature and not exacerbated by straining. There was no associated nausea or visual disturbance. Neurological examination was unremarkable with normal fundi.

Past medical history included laparoscopic surgery in 2014 for painful bowel adhesions. Other long-term medications included oral contraceptive pill. The patient is a non-smoker and occasional consumer of alcohol.

Given the longevity and severity of headaches, extensive investigations were conducted by a neurologist, including a lumbar puncture - which demonstrated normal opening pressure. The headache description was not typical of any particular headache pattern or diagnosis and other causes of headache, such as migraine, medication overuse and idiopathic intracranial hypertension were excluded. There were no mental health issues. Varying medical treatments were trialed to no avail. This included courses of anti-migraine medications, namely pizotifen and topiramate, without symptomatic improvement. Anti-inflammatories and tramadol were also trialed to manage headaches, with little success.

A MRI brain was repeated (Figure $1 \mathrm{~A}-\mathrm{C}$ ), which again demonstrated a stable pineal cyst of benign appearance, measuring $11 \times 20 \times 17 \mathrm{~mm}$ with no significant mass effect or ventriculomegaly.

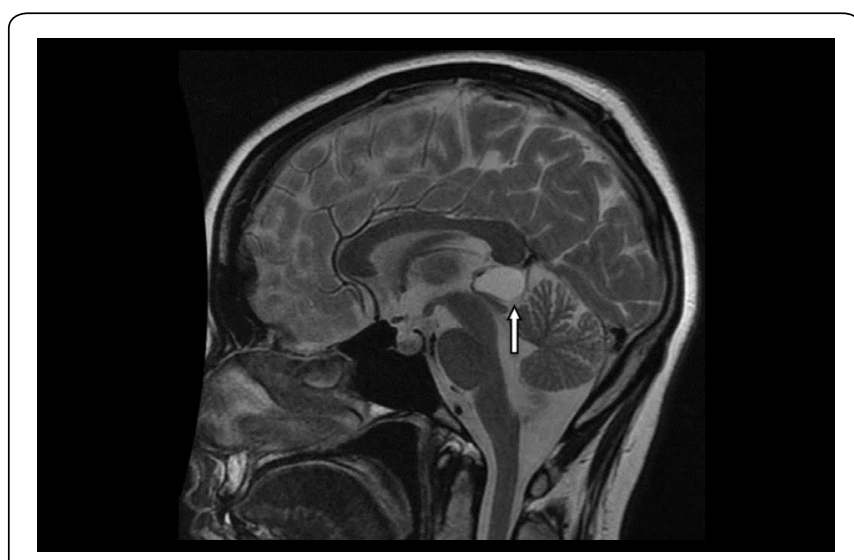

Figure 1A: Pre-operative MRI (1/5/16) with T2 weighting - Sagittal view of pineal cyst measuring $11 \times 20 \times 17 \mathrm{~mm}$ (Arrow).

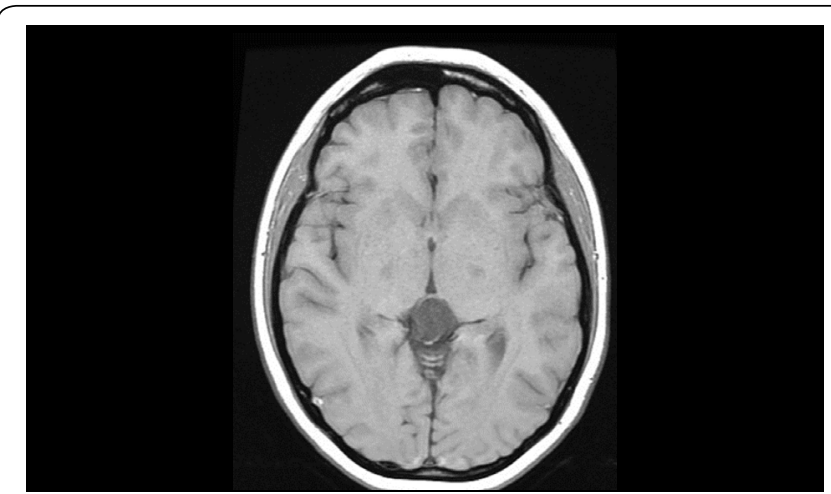

Figure 1B: Pre-operative T1 weighted MRI (1/5/16) - Pineal cyst (Axial view) Note the normal size of the third ventricle.

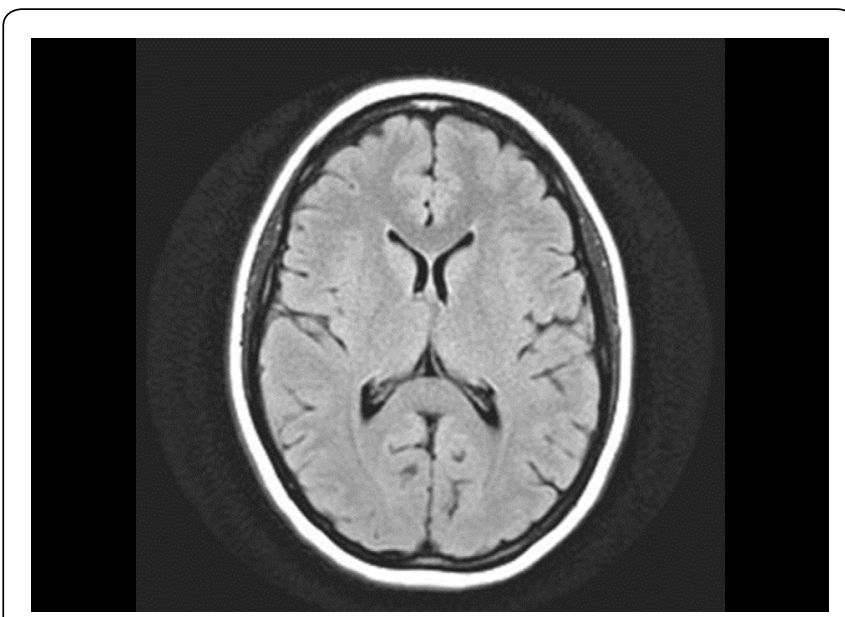

Figure 1C: Pre-operative MRI T1 weighted image (1/5/16). (Axial view) Shows normal sized lateral ventricles.

After extensive discussion, the patient eventually opted for surgical intervention and subsequently underwent posterior fossa craniotomy. She was placed in the prone position and her head was flexed and fixed in the Mayfield 3-pin head rest. Frameless stereotatic guidance was used for trajectory planning and location of the cyst. A posterior fossa craniotomy was performed and the infratentorial supracerebellar approach was used. The major veins were dissected from the posterior aspect of the cyst wall which was then was widely fenestrated. There were no surgical perioperative complications. Histopathology confirmed a pineal cyst with no evidence of malignancy. Immediately post-surgery, the patient reported complete resolution of headache with post-operative MRI brain showed satisfactory decompression of pineal cyst (Figure 2A-C). Upon post-operative review the patient has remained headache free for ten months and remains under surveillance.

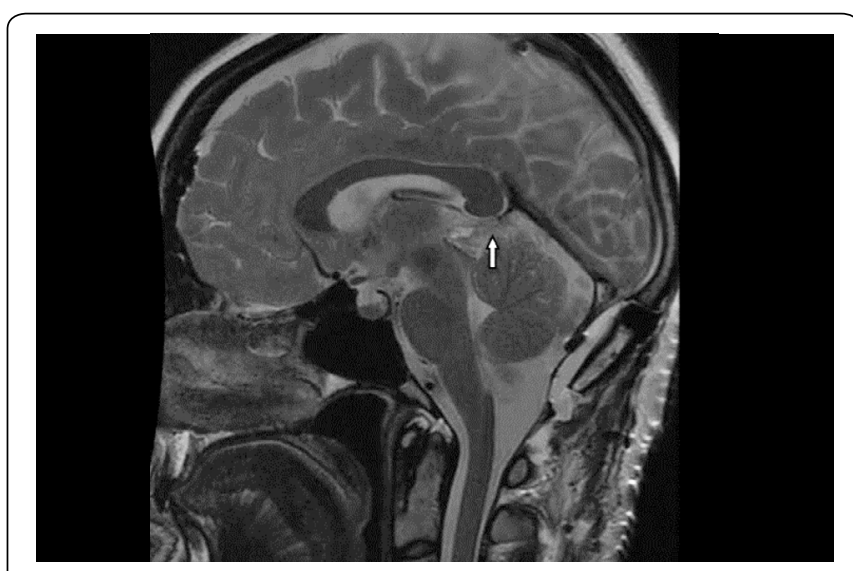

Figure 2A: Post-operative MRI T2 weighed image (4/5/16) - Sagittal view showing successful decompression of pineal cyst (Arrow).

\section{Discussion}

Headaches, particularly migraines, are commonly associated with pineal cysts. Seifert et al. [3] compared 51 cases of pineal cysts with an equal number of controls and reported twice as many headaches in the pineal cyst group (51\% versus $25 \%)$. The headaches associated with pineal cysts may be prolonged, intermittent or acute in duration [4]. 


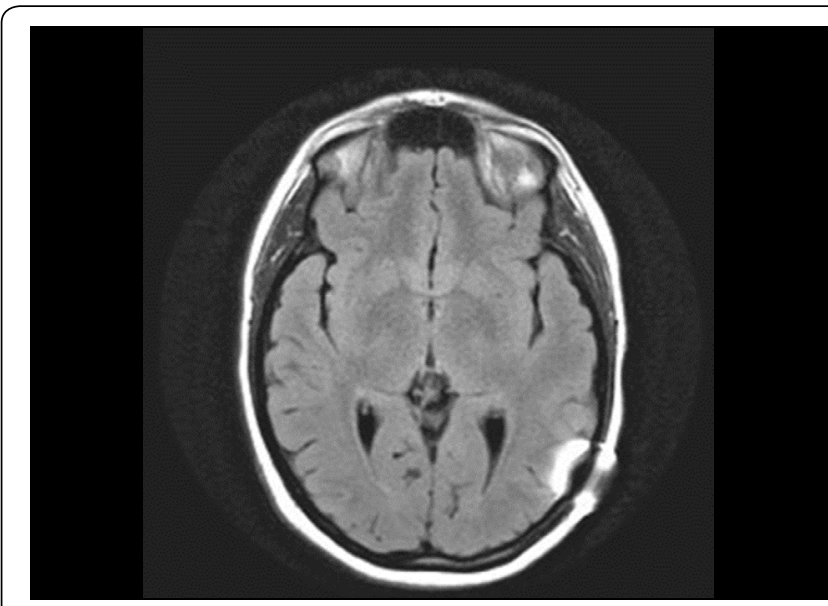

Figure 2B: Post-operative MRI T1 weighted image (4/5/16) (Axial view)showing decompression of the pineal cyst.

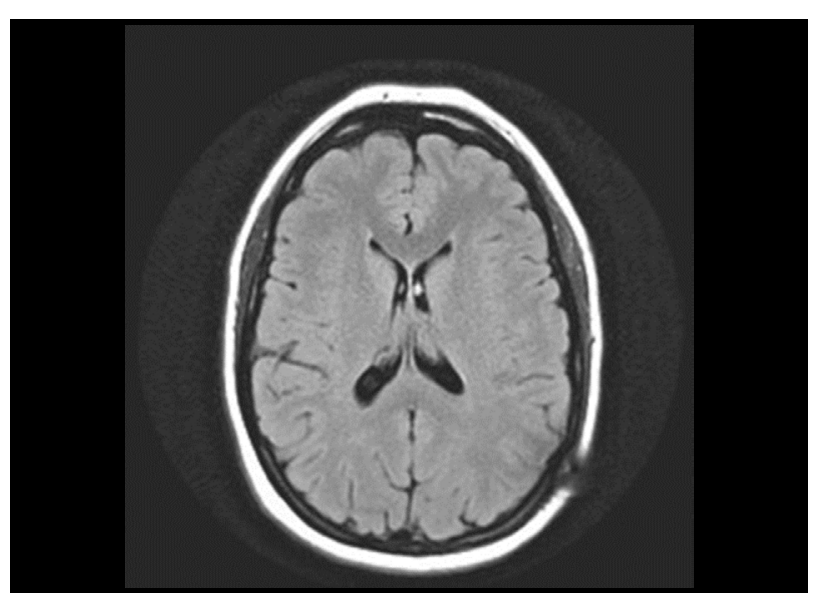

Figure 2C: Post-operative MRI T1 weighted image (4/5/16) (Axial view) showing normal sized Lateral ventricles.

Pathophysiology of headaches associated with pineal cysts remains to be fully determined, but intermittent occlusion of cerebrospinal fluid pathways and abnormal melatonin secretion have been suggested $[5,6]$. Relative venous obstruction due to stretching of the internal cerebral veins may be another mechanism.

Surgical intervention is generally recommended for benign pineal cysts with radiological progression in size and symptomatic patients with secondary hydrocephalus or gaze paresis $[2,5]$. However, indications for surgical management of pineal cysts with non-specific symptoms are poorly defined [5]. In fact, in a recently conducted survey involving 110 neurosurgeons, only $15 \%$ of surveyed neurosurgeons quoted non-specific symptoms, such as headache, as indication for surgery [2].

In a retrospective study conducted by Kalani et al. [5] involving 18 patients who underwent pineal cyst resection in the absence of ventriculomegaly or Parinaud's syndrome, headache was one of the presenting symptoms in 17 patients. At a mean of 19.1 months post-surgical resection of pineal cysts, 94\% of the patients reviewed had improvement or resolution of symptoms, including all patients with headaches. There was no specification that any of these cases presented with the sole symptom of headache. Such findings indicate that surgical intervention may play a role in management of symptomatic pineal cysts in the absence of ventriculomegaly and gaze paresis [5].

The supracerebellar infratentorial approach is the preferred resection technique [2]. However, such an approach may be associated with unpredictable venous drainage and therefore, venous-related complications may occur including: cerebellar swelling, cerebellar venous infarction and venous sinus injury. Other less severe complications may include dizziness, headache and nausea [7]. There needs to be a risk-benefit ratio which takes into account the possibility of such complications arising from resection and measures this against the severity of symptoms and quality of life the patient experiences without surgical intervention.

Most pineal cysts are small and incidental. Our patient had intractable headache with a large pineal cyst, which resolved completely with cyst fenestration. The headache was the sole symptom and occurred in the absence of overt ventriculomegaly or tectal compression. Conservative methods trialed prior to resection were unsuccessful. Given the above, the authors feel that surgical intervention may be considered in patients with chronic headache associated with a benign pineal cyst. However, before surgery is considered, other causes of headache should be excluded or treated. Ventricolmegaly and/or tectal plate compression would add further weight to the decision to operate on these patients.

\section{References}

1. $\mathrm{Pu} \mathrm{Y,} \mathrm{Mahankali} \mathrm{S,} \mathrm{Hou} \mathrm{J,} \mathrm{Li} \mathrm{J,} \mathrm{Lancaster} \mathrm{JL,} \mathrm{et} \mathrm{al.} \mathrm{2007.} \mathrm{High}$ prevalence of pineal cysts in healthy adults demonstrated by highresolution, noncontrast brain MR imaging. AJNR Am J Neuroradiol 28(9): 1706-1709. https://doi.org/10.3174/ajnr.A0656

2. Majovsky M, Netuka D, Benes V.2016. Clinical management of pineal cysts: a worldwide online survey. Acta Neurochir (Wien) 158(4): 663669. https://doi.org/10.1007/s00701-016-2726-3

3. Seifert CL, Woeller A, Valet M, Zimmer C, Berthele A, et al. 2008. Headaches and pineal cyst: a case-control study. Headache 48(3): 448452. https://doi.org/10.1111/j.1526-4610.2007.00965.x

4. Michielsen G, Benoit Y, Baert E, Meire F, Caemaert J. 2002. Symptomatic pineal cysts: clinical manifestations and management. Acta Neurochir (Wien) 144(3): 233-242. https://doi.org/10.1007/ s007010200031

5. Kalani MY, Wilson DA, Koechlin NO, Abuhusain HJ, Dlouhy BJ, et al. 2015. Pineal cyst resection in the absence of ventriculomegaly or Parinaud's syndrome: clinical outcomes and implications for patient selection. J Neurosurg 123(2): 352-356. https://doi. org/10.3171/2014.9.JNS141081

6. Peres MF, Zukerman E, Porto PP, Brandt RA. 2004. Headaches and pineal cyst: a (more than) coincidental relationship? Headache 44(9): 929-930. https://doi.org/10.1111/j.1526-4610.2004.04178_2.x

7. Jakola, AS, Bartek, J, Mathiesen, T. 2013. Venous complications in supracerebellar infratenotiral approach. Acta Neurocbir (Wien) 155(3): 477-478. https://doi.org/10.1007/s00701-012-1614-8 